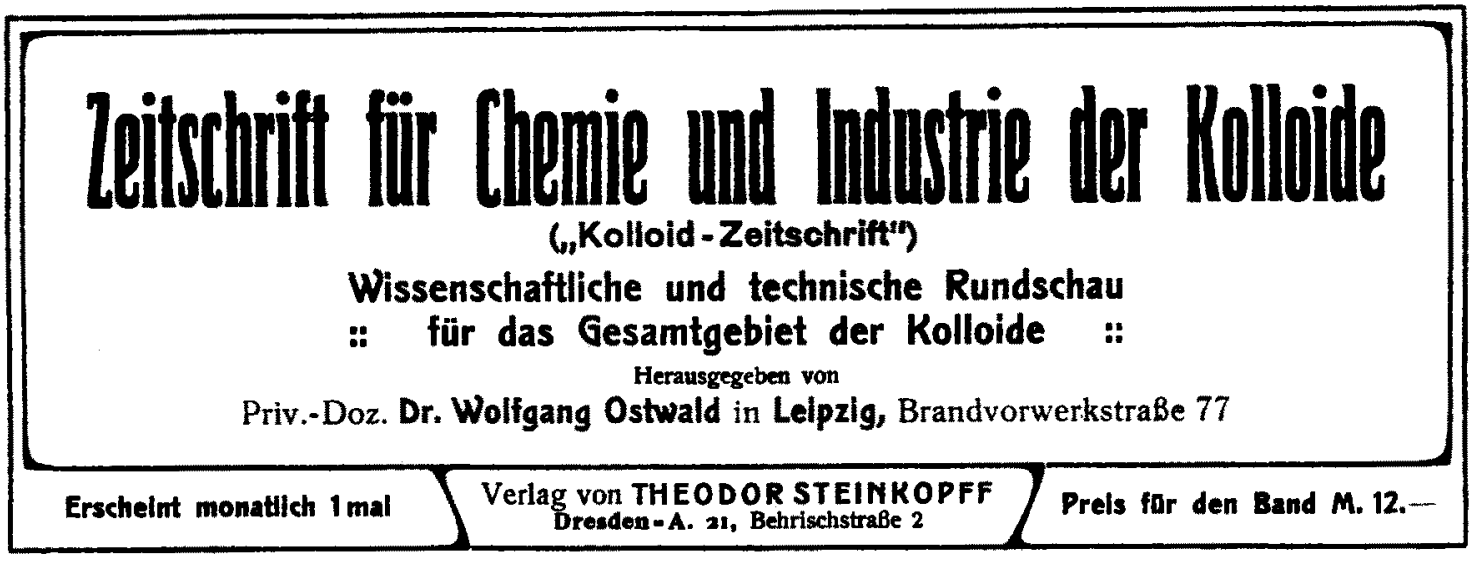

\title{
Einige Beobachtungen über die Waschwirkung der Seifen.
}

\author{
Von W. Spring. Erste Mitteilung.
}

Seit langem hat man sich das Waschvermögen der Seifenlösungen zu erklären gesucht, d. h. die Fähigkeit, die sie besitzen, die Schmutzflecken zu entfernen. Es gehört nun nicht in den Rahmen dieser Notiz, im einzelnen an die verschiedenen Erklärungen zu erinnern, die vorgeschlagen worden sind. Diese finden sich in den Werken gesammelt und besprochen oder in wohlbekannten Sonderartikeln, die von den Seifen und ihren Eigenschaften handeln. - Ich werde mich darauf beschränken, aus diesen Erklärungen mich dessen $z u$ bedienen, was nötig ist, um die Daseinsberechtigung der vorliegenden Arbeit zu erweisen. Bei diesem Anlasse wird $z u$ bemerken sein, dab sie fast alle, wenn nicht überhaupt alle, von einem Punkte ausgehen, dem die Einfachheit fehlt, die eine unmittelbare experimentelle Prüfung erfordert, und daB sie demnach nur eine einstweilige Lösung haben liefern können. In der Tat sehen sie die Schmutzflecken, die die Seife wegnimmt, als einen Ueberzug an, der von einer Mischung von fettigen Stoffen mit Teilchen von verschiedener Natur gebildet wird, und sie befassen sich zunächst damit, ausfindig zu machen, wie sich die Fettkörper in den Seifenlösungen verflüssigen oder auflösen. Die Fortnahme der so befreiten festen Teilchen durch die Flüssigkeitswirbel und durch den Seifenschaum erscheint infolgedessen nur sekundär.

Chevreuil hat als erster eine Erklärung dieser Art zu formulieren versucht. In seinen denkwürdigen Studien über die Fettkörper hatte er angemerkt, daß sich die Seifen in Berührung mit Wasser in saure Salze von geringerer Lösichkeit und in freies Alkali oder in basische Seifen zersetzen. Er sah in dieser Tatsache die soviel gesuchte Erklärung der Waschwirkung der Seife. Nach seiner Ansicht würde das aus der Zersetzung der Seife herrührende Al'sali die Fettstoffe durch Verseifung auflösen, und die entfetteten Teilchen wïrden sich sonach in den sauren Seifen von der Art umkleidet finden, dab sie verhindert wären, sich von $n e$ 'm auf den Gegenständen fest¿ usetzen.

Es ist nicht schwer, einzusehen, dab diese Erklärung fehlerhaft ist; sie stellt sogar einen $\mathrm{Kreis}$ falscher Schlüsse auf. Wenn tatsächlich die aktive Masse des Wassers genügt, um die Seife zu dissoziieren, warum stellt sie sich dann nicht der Verseifung der Fettstoffe entgegen? Wenn man sich andererseits vergegenwärtigt, daß das Verhältnis der durch Wasser dissoziierten Seife außerordentlich klein ist, und daB die Verseifung zu den langsamsten chemischen Vorgängen gehört, begreift man nicht, daß die Waschung in wenigen Augenblicken bewerkstelligt wird. Noch weniger versteht man, dab sie besser in konzentrierterer Seifenlösung geschieht, die dennoch weniger dissozilert ist als eine andere verdünntere.

Noch andere Bemerkungen könnten dazu gemacht werden, aber es ist unnütz, Gewicht darauf zu legen, da die Theorie von Chevreuil von anderen verdrängt worden ist. Eine viel mehr mechanische Erklärung, die von einer gewissen Phantasie nicht frei ist, ist von W.S. Jevons ${ }^{1}$ ) vorgeschlagen worden. Der Autor nimmt im ganzen an, daß der Seifenzusatz zum

2) Chem. Zeitg. 2, 457 (1878). 
Wasser die Oberflächenspannung erhöht und die Brown'sche Bewegung der suspendierten Teilchen heftiger macht. Diese Teilchen würden sich mit Macht auf jene niederschlagen, die den Gegenständen anhaften, sie in Bewegung setzen und forttragen. Fast alle in Wasser löslichen Stoffe würden die Eigenschaft haben, dieser Bewegung Einhalt zu tun; darum, sagt Jevons, eignen sich destilliertes Wasser und Regenwasser am besten zum Waschen. Es ist unnütz, uns bei dieser Erklärung aufzuhalten, die die Härte gewisser Wässer zu wohlfeil preisgibt.

Später hat H.W. Hillger ${ }^{2}$ ) geglaubt, dab er den Grund des Waschvermögens der Seifen in der Fähigkeit suchen müBte, die Fette in Emulsion zu bringen. Die Seifenlösungen vermögen die öligen Gewebe zu benetzen, weich zu machen und so die Wegschaffung der festen Teilchen $z u$ erleichtern. Die Seifenemulsionen hatten schon den Gegenstand einer Studie von F. G. Donnan ${ }^{3}$ ) gebildet, der den Grund ihrer Beständigkeit angegeben und ihre Verwandtschaft mit den Schäumen hervorgehoben hat.

Vor einigen Jahren hat R. Falk ${ }^{4}$ ) eine physikochemische Theorie geliefert, die deswegen interessant ist, weil sie auch von Lösungen in Alkohol Rechenschaft gibt, die, wie bekannt, nicht die Wirksamkeit wässeriger Lösungen haben. Nach des Verfassers Meinung würde man zwei Phasen in der Reinigung betrachten können: die erste würde durch die Konzentration der Seife auf dem Objekt charakterisiert sein, die den Fettkörper lösen würde, und die zweite durch die Wegnahme des Schmutzes infolge der $S c h a u m e r z e u g u n g$ und der Wirkung des Ausspülens. Der wesentliche Punkt in der ersten Phase würde die Betätigung des Adhäsionsvermögens sein, das die gelöste Seife besitzt. Dank ihres Bestrebens, sich auszubreiten, würde die Seifenlösung in alle Zwischenräume der Gegenstände eindringen. Sie würde die Gallerten und Schleime aufquellen lassen und den Austritt der Teilchen bewirken, die sich darin eingeschlossen finden. Die Seifenlösungen in Alkohol, die auch die Fähigkeit haben zu benetzen, wirken in erster Annäherung wie die wässerigen Lösungen, aber sie haben nicht, wie das Wasser, die Eigenschaft, die Häutchen aufzublähen; sie ziehen sie im Gegenteil zusammen und verhärten sie. Weiter geben sie nicht einen so beharrlichen

2) Journ. Am. Chem. Society 25, 511 (1903).

3) Zeitschr. f. phys. Chem. 31, 42 (1899).

4) Zeitschr. f. Elektrochemie 10, 834 (1904).
Schaum. Infolge dieser Tatsachen ist die Reinigung im Alkohol weniger leicht als im Wasser. Die zweite Phase der Reinigung im Wasser beginnt mit der Bildung des Schaums. Die festen Teilchen finden in diesem ein Vehikel, das sie fortführt; infolge des Zustandes ihrer Oberflächenspannung umkleiden die Schäume die Teilchen und nehmen sie weg.

Endlich soll noch erinnert werden, daß Knapp ${ }^{5}$ die Waschwirkung der Seifen dem großen Benetzungsvermögen allen Körpern gegenüber zuschreibt, das diese Lösungen in einem höheren Grade als die anderen Flüssigkeiten besitzen. Die Auflösung der Fettkörper erheischt natürlich eine vollkommene Berührung zwischen dem Lösungsmittel und dem zu lösenden Körper. Das Seifenwasser zieht sich leicht in die Gewebe ein, es gleitet zwischen die Fasern und die Schmutzflecken und nimmt diese fort.

Man sieht, die Autoren dieser verschiedenen Erklärungen scheinen vor allem die Einwirkung der Seifenlösungen auf die Fettkörper betrachtet und das Problem der Reinigung von da an als gelöst angesehen zu haben, wo die Auflösung derselben angenommen werden konnte. Mit einem Worte, sie haben gleich zwei Punkte behandelt, deren Prüfung vielmehr folgeweise stattfinden müßte. Tatsächlich wird die Waschwirkung der Seifen auch in Abwesenheit aller Fettkörper energisch ausgeübt. Um sich von der Sache zu überzeugen, hat man nur die zuvor mit Alkohol gewaschene Haut init einem feinen Pulver von Mangansuperoxyd oder Roteisenstein $z u$ bestreichen und die Leichtigkeit festzustellen, mit der die Reinigung mit Hilfe von Seifenwasser vor sich geht, während das reine Wasser nur eine sehr unvollkommene Wirkung ausübt.

Ich habe also geglaubt, daß die Frage der Mühe wert sei, von neuem geprüft zu werden, aber ich habe sie diesmal rein auf die Wirkung der Seifenlösungen auf die Teilchen absolut von Fetten befreiter fester Körper eingeschränkt.

Ich habe zunächst mit Pulvern von feinem und reinem Kohlenstoff (ausgewaschenem KienruB) gearbeitet, weil der Kohlenstoff am häufigsten in den schmutzigen Stoffen angetroffen wird, da er einer der Bestandteile des Rauches ist, der die Luft der Städte verunreinigt. Alsdann habe ich Kieselsäure, Ton, Eisenoxyd gebraucht; diese Stoffe finden sich auch in den gewöhnlichen Schmutzflecken.

5) Lehrbuch der chem. Technologie 1, 625, und 3. 
Um die Auseinandersetzung der Versuche $\mathrm{zu}$ erleichtern, halte ich es für nützlich, gleich jetzt das erhaltene Ergebnis bekannt zu machen, d. h. die neue Erklärung der Waschwirkung der Seife, die ich mir vorzuschlagen erlaube. Auf diese Art wird man keine Mühe haben, sich in den Versuchen zurecht zu finden, von denen weiterhin die Rede sein wird. In Wahrheit wird es sich in diesem ersten Artikel nur um die Wirkung der Seife auf den KienruB handeln; die Zeit hat mir gefehlt, um das Studium der andern Stoffe $z u$ beenden, und es ist sogar vorauszusehen, dab ich es nicht bald werde zu Ende führen können.

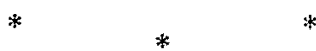

Die Seifenwirkung erklärt sich aus den folgenden Tatsachen:

1. Der Kohlenstoff (KienruB) fördert die Zersetzung einer frischen Seifenlösung in Wasser; er bewirkt oder erleichtert die Bildung eines sauren Salzes, mit dem er sich unter Bildung einer wirklichen Adsorptionsverbindung agglutiniert. Diese widersteht der Einwirkung des Wassers in dem Sinne, dab sie die saure Seife nicht fahren läßt.

Diese Verbindung der sauren Seife mit dem Kohlenstoff ist keine stöchiometrische Verbindung, sondern eine Verbindung, wie sie unter kolloiden Stoffen zustande kommt. Sie läbt sich der vor allem von Ca rey Le a studierten Verbindung vergleichen, die das kolloide Silber mit dem Chlorsilber bildet (das Photohaloid ${ }^{6}$ ), einer Verbindung, die die wahre Substanz des photographischen Bildes ist. Diese Verbindung ist, obwohl sie nur eine Agglutinierung oder eine Adsorptionsverbindung darstellt, nichtsdestoweniger von solcher Beständigkeit, daß sie ihr Chlorsilber nicht mehr sozusagen an eine Lösung von Natriumhyposulfit oder Zyankalium abgibt.

Die Verbindung von Kohlenstoff - Seife bildet sich, weil ihre Bestandteile eine verschiedene elektrische Polarität im Wasser haben. In der Tat, wenn man eine Seifenlösung der elektrischen Kataphorese unterwirft, zersetzt sie sich langsam und man sieht an der Anode weiße Fäden sich sammeln, die saure Seife sind. Die Teilchen von saurer Seife bewegen sich also gegen den elektrischen Strom; sie sind negativ geladen. Dagegen wandert

6) Carey Lea, Kolloides Silber und die Photohaloide. Deutsch von Dr. Luip po-Cramer, Dresden 1908 (Verlag v. Theodor Steinkopff). der im Wasser suspendierte RuB gegen die Kathode. Die Agglutinierung beider Körper fügt sich folglich in die oft schon bei der Agglutinierung der Kolloide beobachteten und besonders sorgfältig von Lottermoser studierten Tatsachen ein.

2. Der trockene oder im Wasser suspendierte $\mathrm{RuB}$ bildet eine mehr oder weniger beständige Adsorptionsverbindung mit den festen Körpern, ganz besonders mit der Zellulose.

Die Tatsache ist unter anderem dadurch bewiesen, daß eine Suspension von Kienruf in Wasser ihren ganzen RuB auf einem Papierfilter zurückläBt und das Filtrat nichts weiter als klares Wasser ist.

Wenn man das Filter in der Weise wendet, daB der KienruB auf die AuBenseite des gebildeten Konus $z u$ liegen kommt und nun zur Waschung schreitet, nimmt das Wasser nichts von dem mit, was am Papier haftet. Die kolloide Verbindung Kohlenst off-Papier wird also nicht von reinem Wasser zerstört.

3. Eine Suspension von Kienrub in Seifenwasser kennzeichnet sich durch ihre Beständigkeit und vor allem dadurch, daB sie gänzlich durch ein Papierfilter durchgeht, ohne etwas von ihrem Kohlenstoff zurückzulassen. Das Papier wird infolge des Filtrierens nicht einmal geschwärzt.

Wenn man überlegt, daß die Teilchen der Verbindung Kohlenst off-Seife notwendig gröber sein müssen als die Teilchen von freiem, nicht mit Seife vereinigtem Kohlenstoff, so wird man annehmen müssen, dab das Filtrieren einer Kohlenstoffsuspension in reinem Wasser in keiner Weise als ein einfaches Durchsieben verstanden werden kann, d. h. als ein Vorgang, der eintreten würde, wenn die Poren des Filters eine kleinere Oeffnung hätten, als den Dimensionen der Kohlenstoffteilchen entspräche. Man wird dagegen zu der Annahme geführt, daß die Kohlenstoffteilchen auf dem Filtrierpapier zurückgehalten werden, weil sie mit diesem eine Verbindung von einer gewissen Beständig. keit bilden.

Nach dieser Voraussetzung wird es sehr leicht sein, die von der Seife ausgeübte Reinigung zu verstehen. Der Mechanismus dieser Operation ist kein anderer, als bei den in der Chemie so häufigen Substitutionserscheinungen. Wenn in der Tat ein beschmutztes Objekt einer Verbindung vergleichbar ist, so wird die Reinigung so betrachtet werden müssen, als ob sie das Ergebnis der Substitution der Seife in diesem Objekte wäre 
Diese Substitution würde allemal dann eìntreten, wenn die Affinität der Seife zum Schmutz gegen die Affinität desselben zu den festen Körpern sich durchsetzt. Mit einem Worte kann die Operation durch die allgemeine SubstitutionsFormel $\mathrm{AB}+\mathrm{C}=\mathrm{AC}+\mathrm{B}$ dargestellt werden, und sie ist immer der Bedingung unterworfen, daß der Schmutz mit der Seife eine beständigere kolloide Adsorptionsverbindung bildet, als die, welche er mit einem gegebenen Körper bildete.

Endlich zersetzt sich die Seife im Alkohol nicht so wie im Wasser in einen basischen und einen anderen sauren Teil. Da die notwendigen Bedingungen für die Bildung einer Adsorptionsverbindung mit dem Kienruß also fehlen, ist es natürlich, dab eine Lösung von Seife in Alkohol nicht so wirksam ist wie eine wässerige Lösung.

$$
* \quad *
$$

Gehen wir jetzt die Versuche durch, die zu den vorstehenden Betrachtungen geführt haben:

\section{A. Ausgangsst offe.}

Die angewendete Seife ist die Medizinalseife gewesen:

Die Lösungen von verschiedenem Titer, die gebraucht worden sind, rührten von der Verdünnung einer durch Auflösen von $20 \mathrm{~g}$ Seife in einem Liter Wasser bereiteten Mutterlösung her; dies war also eine zweiprozentige Lösung.

$\mathrm{Da}$ es, wie gesagt, der Gegenstand vorliegender Arbeit war, sich zu versichern, ob die Waschwirkung der Seifen die Folge ihres Vermögens sei, die Fette in Emulsion zu bringen, oder ob sie wohl auch außer der Gegenwart von Fettstoffen ausgeübt werde, war es wichtig, dem KienruB ölige oder harzige Stoffe, die er stets enthält, bis auf die letzten Spuren zu nehmen, bevor er zu den geplanten Versuchen benutzt werden konnte.

$\mathrm{Zu}$ diesem $\mathrm{Zwecke}$ ist er durch Dekantieren nacheinander mit Alkohol, Aether und Benzol gewascben worden. Nach mehreren Wochen lieb die Waschflüssigkeit noch einen Rückstand bei der Verdampfung übrig. Zur schnelleren Beendigung ist der Kienruß dann in einen Extraktionsapparat gebracht und in der Wärme während mehrerer Tage mit Benzoldampf behandelt worden. Der so entfettete Ru B mischt sich mit Wasser mit einer erstaunlichen Leichtigkeit; er gibt nach dem Umrühren eine schwarze Flüssigkeit, die man für eine kolloide Lösung halten würde, so langsam klärt sie sich in der Ruhe auf.

\section{B. Versuche.}

Es ist zunächst versucht worden, wie sich Lösungen von Seife in Wasser in Berührung mit KienruB verhalten. $\mathrm{Zu}$ diesem $\mathrm{Zwecke}$ sind Seifenlösungen mit abnehmendem Titer in gleichen Intervallen von der Mutterlösung (2 Proz.) an bis zum Titer von $1 / 100$ Proz. bereitet worden. Jede dieser Lösungen ist mit demselben Verhältnis von Kienruß geschüttelt, dann in Ruke gelassen worden im Hinblick auf die Feststellung, ob die Schnelligkeit der $\mathrm{Ab}$ setzung des Rußes in Beziehung zu dem Verhältnis an Seife steht. Zum Vergleich ist auch reines Wasser mit demselben Verhältnis an RuB geschüttelt worden.

Das Ergebnis war überraschend: Der Bodensatz steht nicht in einfacher Beziehung zu dem Titer der Seifenlösung: es gibt ein Optimum der Seifenkonzentration, für die der Ruß in Suspension bleibt. Tatsächlich fällt in der konzentriertesten Lösung (2 Proz.) der Rul fast ebenso schnell nieder wie in reinem Wasser, und in Lösungen, die weniger als $1 / 2$ Proz. enthalten, findet das Absetzen auch statt, obwohl langsam; es ist nötig gewesen, zehn Tage zu warten, damit die Klärung offenbar war. Aber in den Lösungen, die nahe an 1 Proz. enthalten, ist das Absetzen noch nicht nach zwei Monaten beendet. Die Tatsache, daß die Suspensionen fester Körper in Seifenwasser beständiger sind, ist schon beobachtet worden, aber das Vorhandensein eines Optimums der Konzentration für Kienrub ist, soviel ich weif, noch nicht angezeigt gewesen.

Um die eben zitierte Tatsache zu kontrollieren, habe ich zu einem Teile der zweiprozentigen Lösung, die den Ruß fallen ließ, Wasser zugesetzt, so daß ihr Titer auf 1 Proz. gebracht wurde, und habe sie von neuem geschüttelt; diesmal ist der Ruß in Suspension geblieben. Dies beweist also den Einfluß der Konzentration

Es ist zu erwähnen, daß alle Seifenlösungen, die schwarz geblieben sind, weil sie den Kohlenstoff in Suspension hielten, nichtsdestoweniger einen Bodensatz aufwiesen. Der Kohlenstoff hat sich also in zwei Teile getrennt, einen, der suspendiert geblieben ist, und den andern, der sich abgesetzt hat.

Erwägt man, daß die zweiprozentige Lösung, die sich rasch klärt, dennoch viel zäher ist, als die weniger konzentrierten Lösungen, so wird man zu dem Gedanken geführt, daß die angewandten verschiedenen Lösungen vom chemischen Gesichtspunkt aus nicht identisch sind. 
Die Seife, dic sie enthalten, kann mehr oder weniger weitgehend gemäßs dem Verhältnis zum Wasser hydrolysiert sein. Man hat also Ursache darzutun, wie sich eine Suspension von RuB in saurer Lösung und in alkalischer Lösung verhält.

$\mathrm{Zu}$ diesem Behufe habe ich äquivalente Lösungen von Salzsäure und von Kaliumhydroxyd mit einem Titer von 1 Prozent, bzw. 1,5, an bis zum Titer von 1 pro Million bereitet und habe sie mittelst KienruB getrübt. Die sauren Lösungen, selbst die schwächsten, haben sich rasch und gut geklärt, während die alkalischen Lösungen den RuB länger in Suspension gehalten haben als das reine Wasser. Mit Rücksicht darauf, mich zu vergewissern, ob eine Grenze der alkalischen Verdünnung festgesetzt werden könne, für die der Kohlenstoff nicht mehr in Suspension gehalten würde, habe ich die Verdünnung gradweise bis zum Titer von 6 Milliardstel getrieben. Diese äußerste Lösung hat drei Tage gebraucht, um sich annähernd zu klären, während in einem Vergleichsversuche reines Wasser seinen ganzen Kohlenstoff in derselben Zeit hatte fallen lassen. Die Schutzwirkung der Alkalien auf die Kohlenstoffsuspensionen ist also von der Ordnung der Empfindlichkeit chemischer Indikatoren, wie der Lackmus oder das Phenolphtalein.

Ich habe sodann erwiesen, ob die dem Wasser nächsten Alkohole, Methanol und A e th a nol*), auch Seifenlösungen bilden, die ein Optimum der Konzentration darbieten, für das der Ruß länger in Suspension bleibt. Ein positives Ergebnis konnte als neues Kennzeichen der Analogie dieser Körper mit Wasser angesehen werden. Tatsächlich setzt sich der Rub viel rascher in diesen trockenen Alkoholen als in reinem Wasser ab. Mehrere Stunden Ruhe genügen zur Erzielung des Ergebnisses. Andererseits bieten die Seifenlösungen wirklich ein Optimum der Konzentration dar. Dieses ist sogar deutlicher als in den wässerigen Lösungen zu bemerken. Es liegt zwischen den Konzentrationen $1 / 50$ und $1 / 60$ Proz. im Falle des Methanols und um 1/200 Proz. im Falle des Aethanols.

Wie man sieht, verhalten sich die Alkohole wie das Wasser, nur daf ihre Masse 50 oder $200 \mathrm{mal}$ die des reinen Wassers sein muß, damit eine Wirkung von gleicher Ordnung erzielt wird.

Es handelt sich jetzt darum, an die chemische Prüfung der Seifenlösungen zu gehen, die RuB abgesetzt haben, um sich zu überzeugen, ob ) Methyl- und Aethylalkohol. - D. Red. dieser nicht beim Ausfällen Seife mitgerissen hat, was ein Agglutinieren beider Körper beweisen würde.

Zu diesem Ende habe ich die gelöste Seife quantitativ bestimmt durch Verdampfung zur Trockne in einer Platinschale erst auf dem Wasserbad, dann bei $120^{\circ}$ bis zur Gewichtskonstanz. Dazu wurden gleiche Volume $(20 \mathrm{ccm})$ von Seifenlösung angewandt, die nicht mit Rub geschüttelt war, verglichen mit Lösungen, die geschüttelt worden waren, in denen sich aber der RuB vollständig abgesetzt hatte. Es handelt sich also ausschließlich um Lösungen mit dem Titer von ungefähr 2 Proz. -- Ein Filtrieren ist natürlich vermieden worden, um keine Komplikation hineinzubringen. Endlich ist jeder der Verdampfungs-Rückstände zu Asche verbrannt und die Asche ist gewogen worden. Die folgende Tabelle faBt die erhaltenen Resultate kurz zusammen:

\begin{tabular}{|c|c|c|c|}
\hline & $\begin{array}{c}\text { Ver- } \\
\text { dampfungs- } \\
\text { Rürkstand }\end{array}$ & $\begin{array}{l}\text { Gewicht } \\
\text { der } \\
\text { Aschen }\end{array}$ & $\begin{array}{l}\text { Proz. der } \\
\text { Aschen }\end{array}$ \\
\hline 1. Analyse: & & & \\
\hline $\begin{array}{l}\text { Vergleichslösung } \\
\text { Lösung mit Boden- }\end{array}$ & 0,3970 & 0,0740 & 18,64 \\
\hline $\begin{array}{l}\text { satz } \\
\text { II. Analyse: }\end{array}$ & 0,3974 & 0,0760 & 19,13 \\
\hline $\begin{array}{l}\text { Vergleichslösung } \\
\text { Lösung mitBoden- }\end{array}$ & 0,4104 & 0,0750 & 18,27 \\
\hline $\begin{array}{l}\text { satz } \\
\text { III. Analyse: }\end{array}$ & 0,4110 & 0,0760 & 18,49 \\
\hline $\begin{array}{l}\text { Vergleichslösung } \\
\text { Lösung mit Boden- } \\
\text { satz . . . . . }\end{array}$ & 0,4110 & 0,0740 & 18,00 \\
\hline
\end{tabular}

Vergleicht man die Gewichte der Verdampfungsrückstände, so ist es nicht möglich, zu einem sicheren Schluß zu gelangen, dernn ob zwar einmal der Rückstand der abge setzten Lösung schwächer gewesen ist, ist er dagegen in den beiden anderen Fällen größer gewesen als der Rückstand der Vergleichslösung. Es ist möglich, daß diese Lösungen noch ein wenig Kohlenstoff einschlossen, dessen Gegenwart der Beobachtung entgangen ist. Aber wenn man den Prozentsatz der Aschen prüft, gelangt man zu einem sehr deutlichen Ergebnis. Hier hat jedesmal die Lösung, die mit RuB geschüttelt worden ist, einen an Asche reicheren Rückstand hinterlassen als die entsprechende Vergleichslösung. $\mathrm{Da}$ der angewandte RuB durchaus keine Asche hinterlieb 
kann man seiner Gegenwart den festgesetzten Ueberschub nicht zuschreiben und man hat daraus $z u$ folgern, daf die Seifenlösung infolge ihrer Berührung mit dem Ruß eine Zersetzung erlitten hat; sie hat sich in einen mehr sauren Teil geschieden, der sich mit dem Ruß agglutiniert hat, und einen anderen mehr basischen, der in Lösung geblieben ist. Der Beweis der Tatsache wird im folgenden $\mathrm{zu}$ finden sein. Zunächst wollen wir.aber noch sagen, dab auch die Lösungen von Seife in Methanol und in Aethanol vor und nach ihrem Schütteln mit RuB geprüft worden sind. Sie haben eine Tatsache festzustellen erlaubt, die eine gewisse Bedeutung für die Lösung der gestellten Aufgaben hat. Die alkoholischen mit RuB geschüttelten Seifenlösungen lassen bei der Verdampfung einen Rückstand übrig, der etwas weniger Asche gibt. - Wenn also wirklich Zersetzung einer Seifenlösung infolge des Schüttelns mit dem Ruß eintritt, würde es diesmal vielmehr die basische Seife sein, die sich, von dem sich absetzenden RuB mitgerissen, aus der Lösung ausscheiden würde. Hier sind einige Analysendaten zur Stütze dieser Folgerung.

\begin{tabular}{|c|c|c|c|}
\hline & $\begin{array}{c}\text { Ver- } \\
\text { dampfungs- } \\
\text { Rückstand }\end{array}$ & $\begin{array}{l}\text { Gewicht } \\
\text { der } \\
\text { Aschen }\end{array}$ & $\begin{array}{l}\text { Proz. der } \\
\text { Aschen }\end{array}$ \\
\hline $\begin{array}{l}\text { Lösung } \\
\text { in } \mathrm{CH}_{3} \mathrm{OH}\end{array}$ & & & \\
\hline Vergleichsversuch & 0,4012 & 0,0744 & 18,54 \\
\hline 1. Versuch & 0,4000 & 0,0704 & 17,60 \\
\hline 2. Versuch & 0,4032 & 0,0696 & 17,26 \\
\hline in $\stackrel{\text { Lösung }}{\mathrm{C}_{2} \mathrm{H}_{5} \mathrm{OH}^{7} \text { ) }}$ & & & \\
\hline Vergleichsversuch & 0,1788 & 0,0312 & 17,45 \\
\hline 1. Versuch & 0,1800 & 0,0312 & 17,33 \\
\hline 2. Versuch & 0,1782 & 0,0310 & 17,39 \\
\hline
\end{tabular}

Man sieht, daß in keinem Falle das Verhältnis der Aschen größer gewesen ist als in den von den Vergleichslösungen herrührenden Rückständen. Die Lösungen in Aethanol haben sogar zwei so nahe Verhältnisse an Asche geliefert, dab man zu dem Schlusse gelangt, $\mathrm{daB}$ in dieser Flüssigkeit die Zusammensetzung der Seife keine Aenderung erleidet, und daß darin kein Agglutinieren mit dem Kienrub hervorgerufen wird.

7) Mit diesem Alkohol ist eine Lösung nur mit einem Titer von weniget als 1 Proz. bereitet worden, weil die Losung zu 2 Proz. in der Kălte gelierte. Der Alkohol war absolut.
Die Seife verhält sich also in den Alkoholen (besonders in Aethanol) anders als in Wasser. Es ist alsdann nicht überraschend, daB die mit Hilfe alkoholischer Seifenlösungen vorgenommenen Waschungen schwächere Resultate geben als Waschungen mit Seifenwasser.

Der in den Seifenlösungen verschiedener Titer gebildete Bodensatz von Kohlenstoff ist seinerseits geprüft worden.

Dieser Bodensatz hat ein Aussehen, das von dem, welches der RuB in reinem Wasser gibt, besonders absticht. $\mathrm{Er}$ ist nicht wie dieser körnig, mager und leicht wieder in Suspension zu bringen, sondern er ist ölig, zähe, er fliebt wie ein dicker Sirup, kurz, er hat alle Eigentümlichkeiten eines Leimpulvers. Auf ein Papierfilter abgesetzt, läbt er in großen Intervallen schwarze Tropfen durchgehen, und bald arbeitet das Filter nicht mehr; dieser Bodensatz verhält sich also so, wie es eine Tonerdesuspension in Wasser tut.

Ein Versuch, diesen Bodensatz in Rücksicht darauf zu waschen, dab die saure Seife, die ihm anklebt, von ihm entfernt werde, ist vollkommen mißglückt. Schüttelt man ihn mit reinem Wasser, so gelingt es wohl, ihn wieder in Suspension zu bringen, aber alsdann setzt er sich nicht mehr ab. Wie oben gezeigt worden ist, klären sich in der Tat die Seifenlösungen mit einem geringeren Titer als 1 Proz. nicht mehr in der Ruhe auf.

Wird alsdann diese schwarze Flüssigkeit auf ein Filter gegossen, so läbt sich feststellen, $\mathrm{daB}$ sie gänzlich durch das Filter durchgeht in dem Grade, dab dieses nicht e inmal davon geschwärzt bleibt, solange die Konzentration der Seife geringer als ungefähr 1 Proz. ist. Ist die Konzentration höher als 1 Proz., so geht die schwarze Flüssigkeit anfangs noch durch das Filter, dann verstopft sich das Filter allmählich.

Unterwirft man geradeso Suspensionen von RuB in Lösungen verschiedenen Titers von Seife in Methanol oder in Aethanol dem Filtrieren, so sieht man das Filtrieren jedesmal in genügender Weise vor sich gehen: das Filter läßt nur eine klare Seifenlösung durch.

Arbeitet man endlich ebenso mit einer RuBsuspension in reinem Wasser, so bemerkt man ebenfalls, daß das Filter allen Kohlenstoff zurückhält.

Aus diesen Versuchen geht notwendigerweise hervor, daß der Bodensatz von Kohlenstoff Seife (saure Seife) fixiert hat und daß er 
alsdann besondere physikalische Eigenschaft besitzt. DaB er durch ein Filter durchgeht, obwohl er mit Seife verleimt ist, während der mit reinem Wasser gemischte RuB nicht durchgeht, zeigt, daß das Filtrieren nicht einfach mit einem $D u r c h s i e b e n$ verglichen werden kann, bei welchem die Körner, die größer sind als die Löcher des Siebes, allein zurückgehalten werden würden. Man muß ein Agglutinieren der Filtersubstanz mit dem Stoff, der zurückgehalten wird, annehmen, wenn anders nicht das Filter sich nicht als Filter betätigt. Dieses Aggiutinieren kann auf sehr einfache Art an den Tag gelegt werden. Es genügt zu diesem Zwecke, ein Filter, auf dem eben eine Suspension von Kienrub in Wasser filtriert worden ist, so umzuwenden, daß der Belag von Kohlenstoff jetzt auf der AuBenseite des Konus sich befindet, und auf dieses Filter reines Wasser zu gießen. Nur der Kohlenstoff, der nicht unmittelbar die Fasern des Filters berührt, reiBt sich los; so daB das Papier vollständig schwarz bleibt.

Nun bleibt noch zu prüfen, wie sich eine Suspension von RuB in Wasser verhält, wenn man sie einer elektrischen Potentialdifferenz unterwirft, und ferner, wie sich eine Seifenlösung unter denselben Bedingungen verhält. Wie bekannt ${ }^{8}$ ), flo ck e n die kolloiden Lösungen und sogar die Suspensionen unter dem EinfluB des elektrischen Stromes. Ihrer chemischen Natur gemäB wandern die im Wasser suspendierten Teilchen mit dem Strom oder gegen den Strom. Diese Tatsache ist neuerdings die $\mathrm{K}$ a t a phore se genannt worden. Sie hat festzustellen gestattet, dab die Ausflockung, die oft hervorgerufen wird, wenn man zwei kolloide Lösungen mischt, einer Verschiedenheit elektrischer Polarität der Teilchen untergeordnet ist. Es ist nun interessant $z u$ untersuchen, ob das Agglutinieren des Kienrußes mit der Seife einer derartigen Tatsache unterworfen ist.

1. Kataphorese des KienruBes.

Solange der Kienrub in reinem Wasser suspendiert ist, ist die Kataphorese zweifelhaft, wenigstens unter der Einwirkung einer Potential-

8) Siehe meine Untersuchungen über den EinfluB der Elektrizität auf die Absetzung truber Flussigkeiten (Bull. de l'Académie de Belgique 35, $780(1898)$, und $504(1900)$, und vor allem die Arbeiten von Lottermoser (Anorg. Kolloide), von Blllitzer (Zeitschr. f. Elektrochemie 8, 638) und von Biltz (Berl. Ber. 37, 1905). differenz von 8 Volt. Alsdann ist die Stärke des Stromes, der durch die Flüssigkeit geht, zu gering. Aber wenn die Flüssigkeit eben alkalisch gemacht wird (eine Bedingung, die, wie oben gezeigt, die Suspension begünstigt) zur Verminderung ihres elektrischen Widerstandes, so wird der Effekt deutlich: der RuB wandert mit dem Strome. Der Kohlenstoff verhält sich also so, wie wenn er tmit positiver Elektrizität geladen wäre: er sammelt sich um die Kathode herum und setzt sich $a b$.

2. Kataphorese einer Seifenlösung. Unterwirft man eine Seifenlösung mit einem Titer von 2 Proz. der Kataphorese, so bemerkt man nach einigen Stunden die Bildung eines weißen Niederschlages um die Anode herum. Am nächsten Tage ist dieser Niederschlag so reichlich vorhanden, dab er sich auf dem Boden der Flüssigkeit sammelt. Alsdann läBt er sich von der übriggebliebenen Lösung bequem durch einfaches Dekantieren trennen. Vergleicht man das Verhältnis der Aschen, die dieser Niederschlag nach dem Trocknen zurücklä日t, mit dem, welches der Verdampfungsrückstand der dekantierten Flüssigkeit gibt, so überzeugt man sich, daB der an der Anode gebildete Niederschlag eine sauere $S$ e ife ist, während die Flüssigkeit eine basische $S$ e ife in Lösung hält. Tatsächlich hat der Verdampfungsrückstand der dekantierten Flüssigkeit 22,14 Proz. Asche hinterlassen, und der weiße getrocknete Niederschlag nur 16,02 Proz. Das Verhältnis dieser Zahlen, nämlich $\frac{22,14}{16,02}=1,38$, zeigt das Verhältnis der während der Dauer der Kataphorese gebildeten sauren Seife.

Die gegen den Strom wandernde saure Seife erweist sich als negativ geladen; sie erfüllt also die für die Bildung einer Adsorptionsverbindung mit RuB erforderlichen physikalischen Bedingungen. Die Frage, ob die Zersetzung der neutralen Seife in Seifensăure und Seifenbase dem elektrischen Strome oder einfach dem Lösungswasser zuzuschreiben ist, bleibt offenbar ungelöst. Es ist wahrscheinlich, daB hier das Wasser die erste Rolle spielt und daB die Kataphorese nur die Seifensäure um die Anode sammelt, ebenso wie auch der RuB nicht die Seifenlösung zersetzt, sondern das eine Produkt der Hydrolyse, die Seifensäure, in Beschlag nimmt.

Ich füge zum Schlusse hinzu, daß ich auch eine Suspension von RuB in einer einprozentigen 
Seilenlösung der Kataphorese unterworfen habe in der Hoffnung, die Agglutinierung sich wieder auflösen $z u$ sehen, d. h. zu sehen, daß der Kohlenstoff die Kathode erreicht und die Fäden der weiBen Seifensäure sich an der Anode anhäufen. Das Ergebnis hat meiner Erwartung nicht entsprochen: die Flüssigkeit hat sich auf einmal an beiden Polen geklärt, und nach zwei Tagen war aller RuB ausgeflockt.
Dies sind die Tatsachen, aus denen ich geglaubt habe die im Verlaufe dieser. Note zusammengefaßten Schlüsse ziehen zu können.

In einem nächsten Artikel werde ich mich mit der Art und Weise befassen, nach der sich Kieselsäure, Eisenoxyd usw. in den Seifenlösungen verhaiten.

Januar 1909.

Lüttich, Institut für Algemeine Chemie.

\title{
Ueber die Existenz und Eigenschaften disperser Systeme im Grenz- gebiet zwischen den kolloiden und kristalloiden Lösungen.
}

\author{
Von The Svedberg, Erste Mitteilung.
}

Für die Erforschung der Eigenschaften disperser Systeme ist es von groBer Wichtigkeit, solche Gebilde zu finden und zu studieren, deren Dispersität zwischen denjenigen der kolloiden und molekulardispersen Systeme liegt.

Bestimmte Methoden für die Herstellung derartiger Lösungen fehlen noch, nur einzelne allgemeine Bedingungen, durch deren Innehalten sie möglicherweise zu erhalten wären, sind bekannt. So hat man $z$. B. mehrmals beobachtet, dab die Homogenität kolloider Gebilde, welche durch chemische Reaktionen in Lösungen entstehen, mit steigender Verdünnung der reagierenden Stoffe inmer größer wird. Da jedoch die Konzentration der dispersen Phase bei einer solchen Steigerung der "Reaktionsverdünnung “ immer kleiner wird und da die Intensitäten der Eigenschaften disperser Systeme mit wachsender Verdünnung der dispersen Phase im allgemeinen gegen Null abfallen, so ist es von vornherein klar, daf bald eine Grenze erreicht wird, über die hinaus ein experimenteller Beweis für die Existenz der eventuell entstandenen dispersen Systeme nicht mehr erbracht werden kann. Durch diesen Umstand werden also die erhaltenen Zahlenwerte der Intensität einer studierten Eigenschaft beim tieferen Eindringen in das interessante Gebiet disperser Systeme extremer Verdünnung immer unsicherer bis zur vollständigen Unbrauchbarkeit.

Betreffs der molekulardispersen Systeme großer Verdünnung wissen wir, daf es in einer Menge von Fällen gelungen ist, Funktionen der gemessenen Eigenschaften zu definieren, die mit der Abnahme der Intensität der gemessenen Eigenschaft, d. h. mit steigender Verdünnung der L.ösung, sich einen konstanten Grenzwert asymptotisch nähern. Bei den Systemen ge- ringerer Dispersität, den kolloiden Lösungen, ist dies aber bisher nicht gelungen und wird wohl im allgemeinen nicht gelingen können, denn aller Wahrscheinlichkeit nach werden diese Grenzwerte hier erst in einem Gebiete sehr nahe an den entsprechenden molekulardispersen Systemen erreicht. Der Kolloidchemiker muB also darauf bedacht sein, Versuche zu machen, um solche Bedingungen aufzufinden, die die Intensitätsabnahme der Eigenschaft durch andere Faktoren zu kompensieren gestatten.

In dieser ersten Mitteilung werde ich den experimentellen Existenzbeweis für einige in dieses Grenzgebiet fallende disperse Systeme führen, deren disperse Phase aus metallischem Gold, aus Eisensesquioxyd und aus Arsensesquisulfid besteht, und über einige optische Eigenschaften derselben berichten.

Für die Herstellung der untersuchten Systeme diente als leitendes Prinzip die schon erwähnte allgemeine Beobachtung, daß die Teilchengröße einer kolloiden Lösung mit steigender Verdünnung bei der Herstellung sich verkleinert. Die studierte Eigenschaft war die Lichtabsorption. Um dieselbe auch in hohen Verdünnungen beobachten zu können, wurde eine Kompensationsmethode benutzt.

Die Lichtabsorption in Lösungen gehorcht bekanntlich (in erster Annäherung) dem Beerschen Gesetz:

Konzentration $\times$ Schichtdicke $=$ Konst., das von großer, bisher fast unbeachteter experimenteller Tragweite ist. Führen wir für den Wert der Konstante dieser Gleichung, bei dem eine Absorption eben noch sichtbar ist, die Bezeichnung lim $\mathrm{k}$ ein und setzen wir ferner die Konzentration in Normalität gerechnet $=c$, die 